Classification

Physics Abstracts

$73.60-78.65$

\title{
Compared optical properties of noble and transition metal-dielectric granular films
}

\author{
S. Berthier $\left(^{*}\right)$ and J. Lafait \\ Laboratoire d'Optique des Solides $\left({ }^{+}\right)$, Université Pierre et Marie Curie, \\ 4, place Jussieu, 75252 Paris Cedex 05, France
}

(Reçu le 27 juin 1985, accepté le 2 octobre 1985)

\begin{abstract}
Résumé. - Les milieux inhomogènes métal-diélectrique présentent des propriétés physiques différentes suivant que l'inclusion métallique est un métal noble ou un métal de transition. Nous présentons une étude comparée de couches minces de $\mathrm{Au}-\mathrm{MgO}$ et $\mathrm{Pt}_{-} \mathrm{Al}_{2} \mathrm{O}_{3}$ (préparées par pulvérisation cathodique) à partir de leur propriétés optiques, électriques et de leur structure. L'analyse des propriétés optiques est réalisée à l'aide de deux théories de milieu effectif : Maxwell Garnett et Bruggeman. Les deux systèmes percolent pour des fractions volumiques en métal élevées $(0,4-0,5)$. Ils différent essentiellement par l'anomalie diélectrique et le rôle joué par le temps de relaxation des électrons de conduction du métal sur les propriétés optiques du milieu granulaire.
\end{abstract}

Abstract. - Metal-dielectric inhomogeneous media exhibit contrasted physical properties according to the nature of the metallic inclusion : noble or transition. A comparison between $\mathrm{Au}-\mathrm{MgO}$ and $\mathrm{Pt}-\mathrm{Al}_{2} \mathrm{O}_{3}$ thin films (prepared by sputtering), based on their optical, electrical and structural properties, is presented. The optical properties are analysed using two effective medium theories : Maxwell Garnett and Bruggeman. Both systems percolate at high metal volume fraction (0.4-0.5). They mainly differ in the dielectric anomaly and the influence of the conduction electron relaxation time on the optical properties of the inhomogeneous medium.

\section{Introduction.}

The metal dielectric inhomogeneous media are among the most interesting materials of the last ten years. Their interest lies in their numerous applications to many disciplines, and more specifically to physics. Their optical (high frequency) and electrical (zero frequency) properties show large variations as a function of the volume fraction, the size and shape and the statistic distribution of metallic inclusions. These variations are of particular interest around the critical metallic volume fraction $q_{\mathrm{c}}$ corresponding to the electrical percolation. One also observes for values of the order of $q_{\mathrm{c}}$ an optical cross-over where a transition occurs between a dielectric and a metallic behaviour. Moreover, at constant volume fraction (in a large fraction range) the dielectric function, as a function of frequency, exhibits a resonance, the socalled dielectric anomaly.

Our aim, in this paper, is to show how these properties depend on the nature of the metallic inclusion in the compound : noble metal or transition metal. The

(*) Also : Université de Corse, BP 24, 20250 Corte, France.

$\left({ }^{+}\right)$U.A. au CNRS no 040781. discussion is based on experimental results on Au$\mathrm{MgO}$ and $\mathrm{Pt}-\mathrm{Al}_{2} \mathrm{O}_{3}$ thin films prepared by co-sputtering. Their optical properties, electrical properties and structures are compared. The dielectric function deduced from optical measurements is analysed using two effective medium theories derived from the Maxwell Garnett [1] and Bruggeman [2] approaches, the efficiencies of which are eventually compared.

Regarding the electronic structure $\mathrm{Au}$ [3] and Pt [4] and more generally noble and transition metals essentially differ in the position of the Fermi level with respect to the $d$ band. In Au the d-band is completely filled, whereas $E_{\mathrm{F}}$ cuts the d-band in Pt. Consequently, the absorption edge, characteristic of the interband transition onset, which is clearly visible in the imaginary part of the dielectric function, is situated around $2.45 \mathrm{eV}$ in $\mathrm{Au}$ while it is shifted towards much lower energies (around $0.1 \mathrm{eV}$ ) in Pt. In other words, the near infrared behaviour of the dielectric function is essentially governed by intraband transitions of the conducting electrons in Au and still largely dominated by interband transitions in Pt. These well-known considerations will be important in the discussion of the optical properties of the granular films. Section 2 is devoted to the preparation and the characterisation 
of the $\mathrm{Au}-\mathrm{MgO}$ and $\mathrm{Pt}-\mathrm{Al}_{2} \mathrm{O}_{3}$ thin films. In section 3, the optical properties are discussed and analysed with the two effective medium models.

\section{Preparation and characterization of the inhomo- geneous films.}

2.1 Preparation. - The inhomogeneous thin Au$\mathrm{MgO}$ and $\mathrm{Pt}-\mathrm{Al}_{2} \mathrm{O}_{3}$ thin films were prepared with neighbouring but slightly different techniques.

$\mathrm{Au}-\mathrm{MgO}$ [5] films were deposited by reactive cosputtering (Magnetron source) onto optically polished glass substrates. The target is a rectangular magnesium foil with circular holes of diameter $3 \mathrm{~mm}$. The holes are filled with gold cylinders. The composition of the film can thus be varied over a wide range according to the number of holes in the target (24-80). Three substrates can be placed at $6 \mathrm{~cm}$ in front of the target on a doubly rotating system. Each substrate rotates on its own axis at $32 \mathrm{rev}$. $\min ^{-1}$, to ensure that the film thickness is uniform, and the three substrates rotate about the axis of the chamber at $11 \mathrm{rev} . \mathrm{min}^{-1}$, to homogenize the composition of the deposit. The substrates can be heated to $200^{\circ} \mathrm{C}$. The sputtering conditions are as follows : argon pressure, $5 \times$ $10^{-3}$ torr; oxygen pressure, $2 \times 10^{-3}$ torr; rate of deposition, $35-135 \AA \mathrm{min}^{-1}$ (depending on the target and the substrate temperature).

Pt- $\mathrm{Al}_{2} \mathrm{O}_{3}$ [6] films were deposited onto optically polished glass by r.f. cosputtering. The target consists in an $\mathrm{Al}_{2} \mathrm{O}_{3}$ disc $(13 \mathrm{~cm}$ in diameter) with circular holes of diameter 5 min disposed in a hexagonal array. The holes can be filled with platinum pellets. The composition of the film can be varied over a wide range according to the number of platinum pellets in use. The substrates are located on a rotating sample holder. The films obtained by this rotating sample technique are homogeneous in composition and uniform in thickness. The rate of deposition is $50 \mathrm{~A} \mathrm{~min}^{-1}$ and the residual pressure of argon is $7 \times 10^{-3}$ torr.

\subsection{Characterization. - The thickness of the films} with a low metallic volume fraction was determined by $\mathrm{X}$-ray interference under grazing incidence. For $\mathrm{Au}$ or $\mathrm{Pt}$ volume fractions greater than 0.35 this technique failed, due to the scattering of the X-rays by the numerous metallic inclusions. A talystep mechanical step height monitor was then used.

The composition of the deposits (metallic volume fraction $q$ ) was determined for both compounds by electron microprobe analysis. In addition, chemical analysis and ESCA were performed on $\mathrm{Au}-\mathrm{MgO}$ samples. In spite of the relatively poor accuracy of some of these methods, the results are in fairly good agreement.

The D.C. resistivity of the films was deduced from resistance measurements using an electrometer (before percolation) or a resistance bridge (near and after percolation).
The results of these characterizations on different samples are summarized in tables Ia et Ib.

Table I. - Characteristics of the $\mathrm{Pt}_{-} \mathrm{Al}_{2} \mathrm{O}_{3}$ (Ia) and $\mathrm{Au}-\mathrm{MgO}(\mathrm{Ib})$ thin granular films and fitted values of $q$ (metal volume fraction) and $L$ (depolarization factor) using M.G. and BR Theories.

a)

\begin{tabular}{|c|c|c|c|c|c|c|c|}
\hline \multicolumn{3}{|c|}{$\begin{array}{l}\mathrm{Pt}_{-\mathrm{Al}_{2} \mathrm{O}_{3}} \\
\text { sample }\end{array}$} & $\begin{array}{l}1 \\
+\end{array}$ & $\begin{array}{l}2 \\
\times \\
\end{array}$ & $\begin{array}{l}3 \\
0\end{array}$ & $\begin{array}{l}4 \\
\Delta\end{array}$ & $\begin{array}{l}5 \\
\mathbf{a}\end{array}$ \\
\hline \multicolumn{3}{|c|}{ thickness (l) } & 790 & 870 & 590 & 375 & 504 \\
\hline \multicolumn{3}{|c|}{ resistivity $(\Omega, m)$} & 39 & 0.1 & $3.410^{-4}$ & $1.110^{-4}$ & $0.210^{-4}$ \\
\hline \multicolumn{3}{|c|}{$\begin{array}{l}\text { Pt volume } \\
\text { fraction }\end{array}$} & 0.18 & 0.31 & 0.34 & 0.43 & 0.53 \\
\hline \multirow{4}{*}{ 嵒 } & \multirow{2}{*}{$\begin{array}{r}\text { FIT of } \\
\varepsilon_{1} \text { and } \varepsilon_{2}\end{array}$} & $q$ & .138 & .216 & .217 & .298 & .122 \\
\hline & & $L$ & .296 & .282 & .262 & .306 & .050 \\
\hline & \multirow{2}{*}{$\begin{array}{r}\text { FIT of } \\
R \text { and } T\end{array}$} & $q$ & .14 & .20 & .244 & .341 & .237 \\
\hline & & $\mathbf{L}$ & .299 & .254 & .301 & .370 & .217 \\
\hline ن & $\begin{array}{l}\text { FIT of } \\
\varepsilon_{1} \text { and } \varepsilon_{2}\end{array}$ & $\mathbf{q}$ & 0.091 & 0.078 & 0.103 & 0.134 & 0.07 \\
\hline
\end{tabular}

b)

\begin{tabular}{|c|c|c|c|c|c|c|c|}
\hline \multicolumn{3}{|c|}{$\begin{array}{l}\text { Au-MgO } \\
\text { sample }\end{array}$} & $\stackrel{t}{1}$ & $\begin{array}{l}x \\
2\end{array}$ & $\stackrel{\circ}{3}$ & $\begin{array}{l}\Delta \\
4\end{array}$ & $\begin{array}{l}a \\
5\end{array}$ \\
\hline \multicolumn{3}{|c|}{ thickness ( $\mathbf{\AA})$} & 1014 & 769 & 821 & 717 & 626 \\
\hline \multicolumn{3}{|c|}{ resistivity $\left(\Omega_{m}\right)$} & $\infty$ & $\infty$ & 31 & \begin{tabular}{|l|l|} 
& \\
&
\end{tabular} & 1.2 \\
\hline \multirow{2}{*}{\multicolumn{2}{|c|}{$\begin{array}{l}\text { Au volume } \\
\text { fraction }\end{array}$}} & MPA & 0.16 & 0.26 & 0.28 & 0.31 & 0.38 \\
\hline & & C.A & 0.26 & 0.3 & $.3-.4$ & $.3-.4$ & 0.41 \\
\hline \multirow{2}{*}{ 畕 } & FIT of & q & 0.077 & 0.17 & 0.213 & 0.251 & 0.337 \\
\hline & $\varepsilon_{1}$ and $\varepsilon_{2}$ & $\mathrm{~L}$ & 0.296 & 0.418 & 0.45 & 0.487 & 0.554 \\
\hline$\stackrel{\dot{0}}{\dot{z}}$ & $\varepsilon_{1 \text { and }}^{\text {PT } \varepsilon_{2}}$ & q & 0.314 & 0.441 & 0.491 & 0.525 & 0.579 \\
\hline
\end{tabular}

Electrical percolation. - The plot of the resistivity versus the metallic volume fraction (Fig. 1) clearly shows that the electrical percolation occurs for the

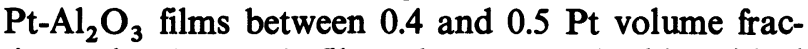
tion. The Au-MgO films do not reach this critical threshold but follow an analogous behaviour for low metal concentration. This high value of the critical volume fraction is characteristic of a $2 \mathrm{D}$ behaviour predicted by Monte Carlo simulations for spherical clusters [7] rather than the $3 \mathrm{D}$ behaviour which is expected. At least, at low volume fraction, it is indeed clear that the Euclidian dimension of the film is 3, due to the relatively high thickness of these films compared to the metallic grain size, the grain shape being roughly spherical. As the percolation is approa- 


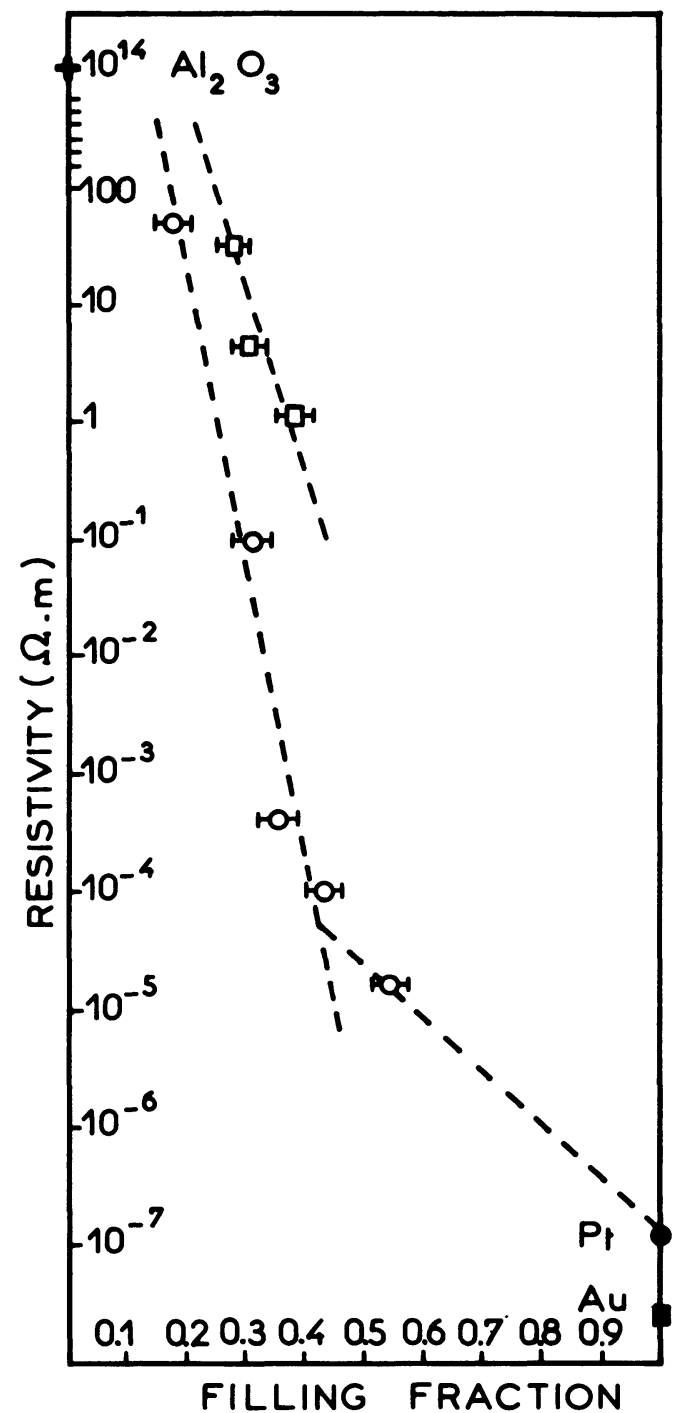

Fig. 1. - D.C. electrical resistivity of $\mathrm{Pt}_{-} \mathrm{Al}_{2} \mathrm{O}_{3}$ and $\mathrm{Au}-$ $\mathrm{MgO}$ cermet films versus the metal volume fraction $q$.

ched, two phenomena conjugate for moving the threshold towards higher metal fraction : 1) the cluster size is increasing, pushing the medium to a 2D behaviour ; 2) the morphology of the samples is such that, up to high metal fractions, each metal grain is surrounded by a layer of the amorphous dielectric matrix (see below); the inhomogeneous medium is not therefore truly random, as already emphasized [8].

The morphology of these films was studied by using transmission electron microscopy (TEM) and electron diffraction performed on thinner films deposited under identical conditions onto carbon coated copper grids (Figs. 2 and 3). The diffraction patterns show that for both compounds, the metallic phase is crystallized whereas the dielectric one is amorphous. Below the percolation threshold, both compounds are composed of roughly spherical metallic inclusions embedded

Fig. 3. - Electron diffraction pattern (3a) and transmission electron micrograph (3b) of a $\mathrm{Pt}_{-} \mathrm{Al}_{2} \mathrm{O}_{3}$ cermet film. (Pt volume fraction $q=0.43$.)
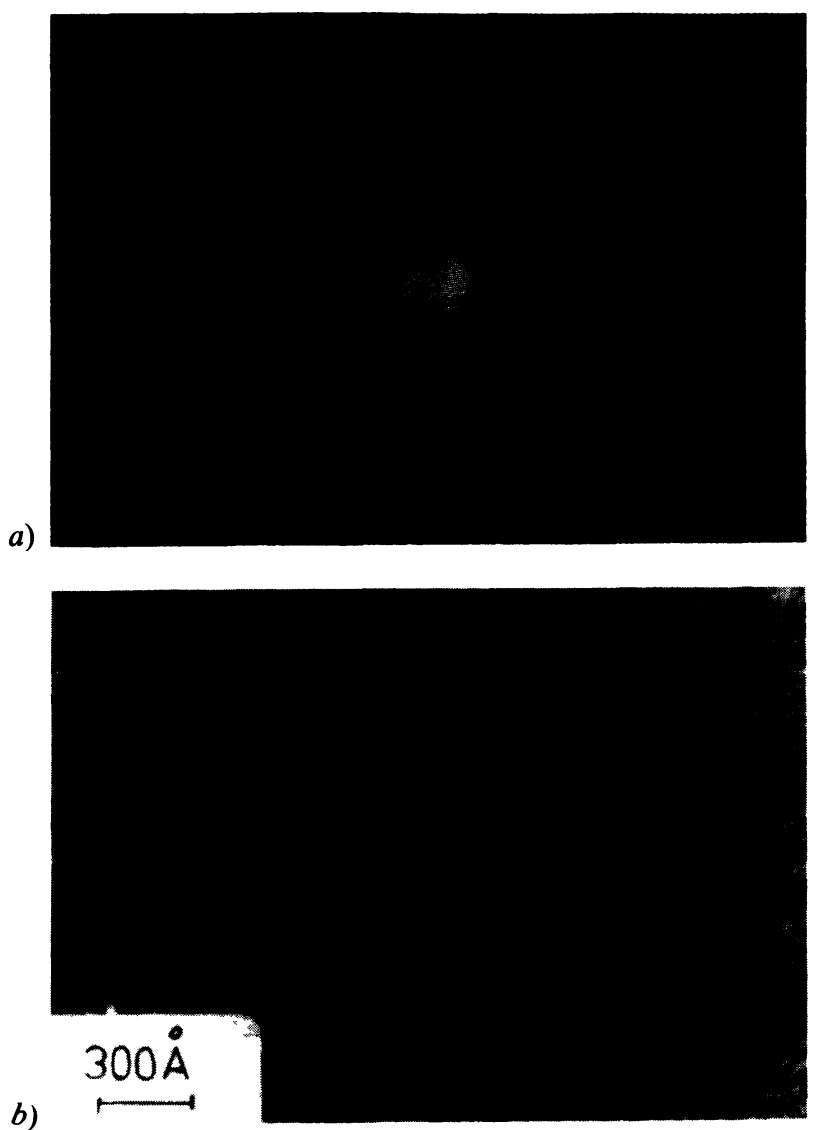

Fig. 2. - Electron diffraction pattern (2a) and transmission electron micrograph (2b) of a $\mathrm{Au}-\mathrm{MgO}$ cermet film. (Au volume fraction $q=0.2$.)

a)

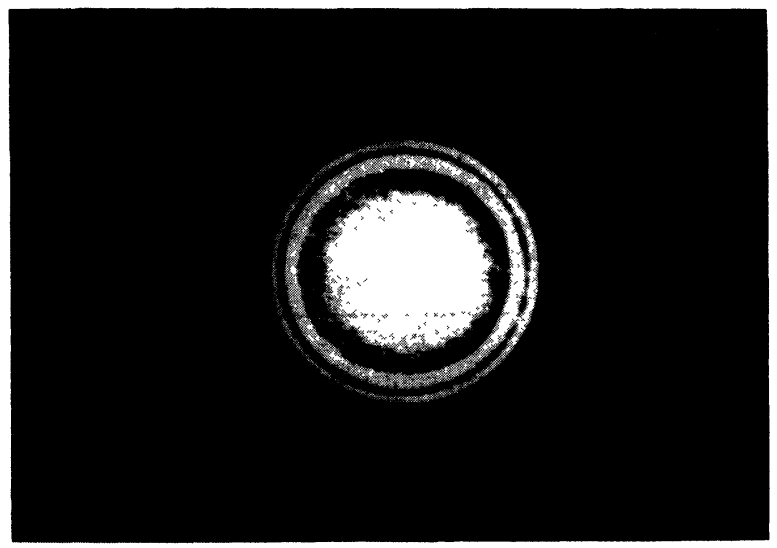

b)

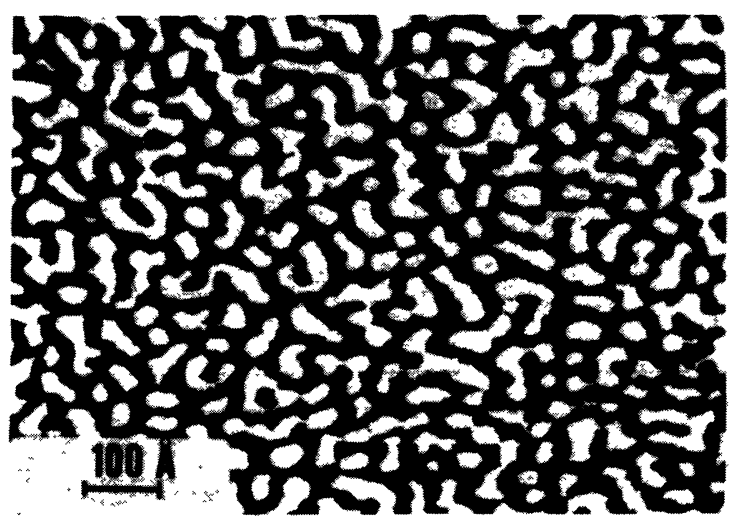


in an amorphous matrix. The metallic cluster mean size is fairly concentration independent (in this dielectric regime) : 4-5 nm for $\mathrm{Pt}$ and 3-5 $\mathrm{nm}$ for $\mathrm{Au}$; but it is increasing with the substrate temperature during the deposition. Around the percolation, the grains tend to become tortuous cluster, the metal and dielectric phases presenting then the same morphology. At the percolation threshold, an infinite cluster is formed throughout the film and a statistical study of its morphology in the framework of scaling laws and fractal theory allows the determination of the
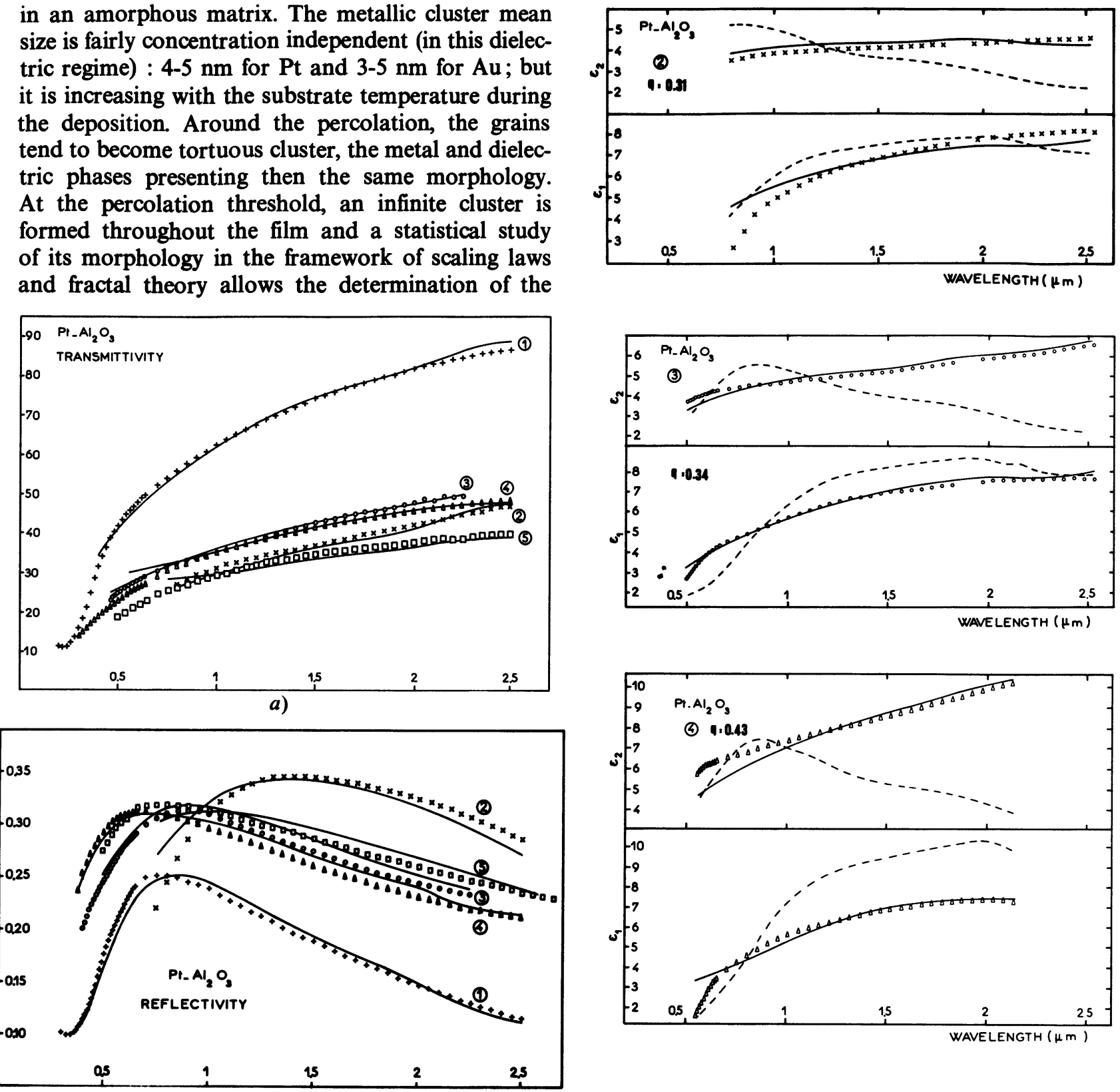

Fig. 4. - Measured transmittivity (4a) and reflectivity (4b) of $\mathrm{Pt}_{-} \mathrm{Al}_{2} \mathrm{O}_{3}$ granular thin films of various thicknesses and metal volume fractions (see Table I) in the 200-3 $000 \mathrm{~nm}$ spectral range. $(1 . q=0.18+, 2 . q=0.31 \times, 3 . q=0.34 \mathrm{O}$, 4. $q=0.43 \Delta$, 5. $q=0.53 \square$.) Fit using BR theory (full lines).
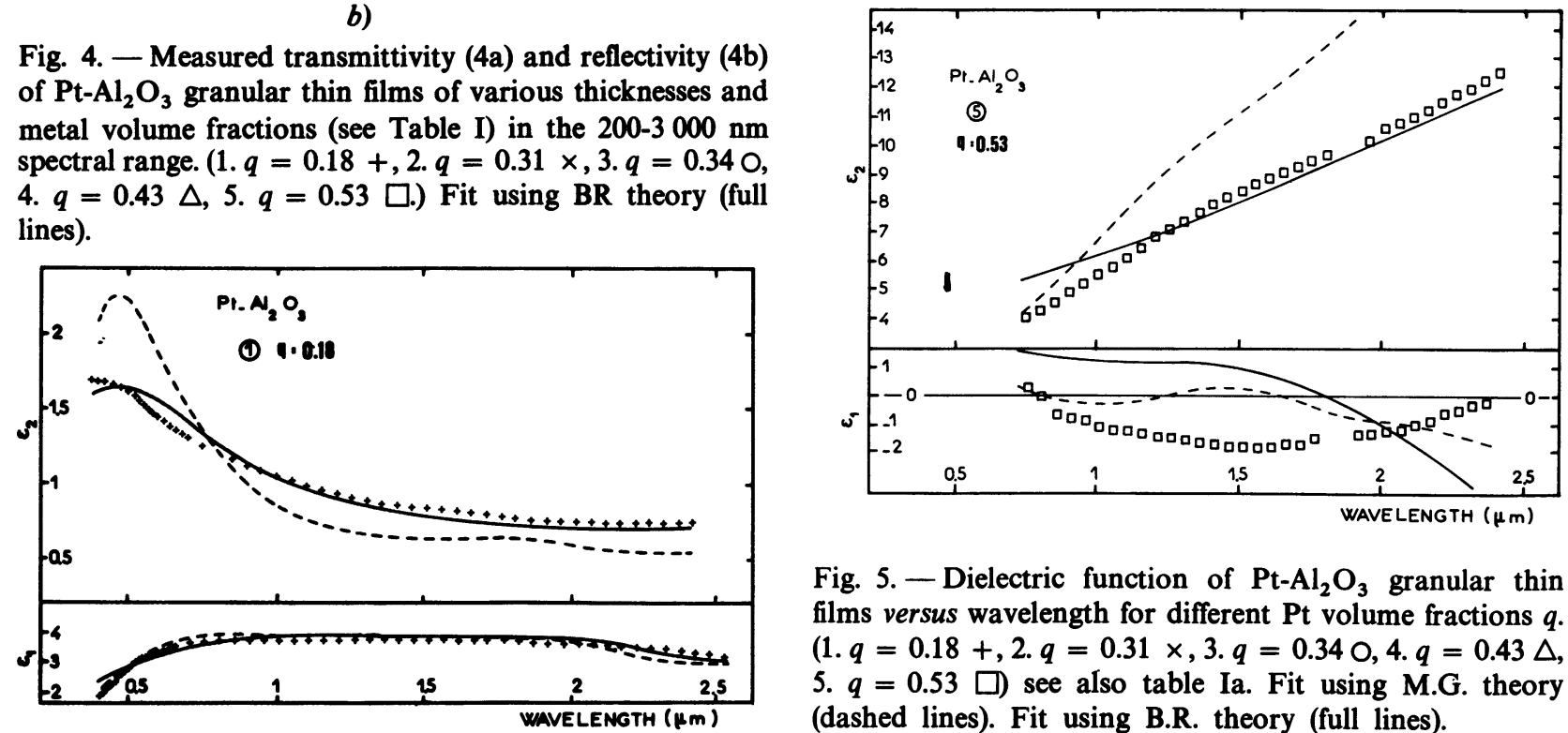

Fig. 5. - Dielectric function of $\mathrm{Pt}-\mathrm{Al}_{2} \mathrm{O}_{3}$ granular thin films versus wavelength for different $\mathrm{Pt}$ volume fractions $q$. $(1 . q=0.18+, 2 . q=0.31 \times, 3 . q=0.340,4 . q=0.43 \Delta$, 5. $q=0.53 \square$ ) see also table Ia. Fit using M.G. theory (dashed lines). Fit using B.R. theory (full lines). 
critical exponents at percolation, the fractal dimension and the percolation correlation length. These results obtained by image treatement, digitalization and binarization will be published elsewhere.

\section{Optical properties.}

3.1 OPTICAL MEASUREMENTS AND DIELECTRIC FUNCTION CALCULATION. - The specular transmittivity $(T)$ and reflectivity $(R)$ of the films were measured under near normal incidence, in the spectral range 300 to $3000 \mathrm{~nm}$ using a Cary 17 spectrophotometer. (For $\mathrm{Pt}_{-} \mathrm{Al}_{2} \mathrm{O}_{3}$ see Figs. 4.)

The complex dielectric function $\tilde{\varepsilon}$ of the films was calculated by solving the implicit system of the two Fresnel's formulae describing $R$ and $T$ as functions of the complex index $\tilde{n}$, the thickness $d$ and the wavelength $\lambda$ :

$$
\text { (1) }\left\{\begin{array}{l}
R=f(n, k, d, \lambda) \\
T=g(n, k, d, \lambda)
\end{array} \quad \tilde{\varepsilon}=\tilde{n}^{2}\left\{\begin{array}{l}
\tilde{n}=n-i k \\
\tilde{\varepsilon}=\varepsilon_{1}+i \varepsilon_{2} .
\end{array}\right.\right.
$$

A computer program for a non-linear least square fit with weighted gradient convergence was used [9].

For several films, no solutions to the equation system (1) has been found below $500 \mathrm{~nm}$. The interesting effects appearing at higher wavelength, this lack is however not too dramatic. It is still possible to analyse the reflectivity and transmittivity spectra directly; the conclusions of both types of treatments are usually in reasonable agreement. The results of $\varepsilon_{1}$ and $\varepsilon_{2}$ are plotted in figures 6 for $\mathrm{Au}-\mathrm{MgO}$ and figures 5 for $\mathrm{Pt}-\mathrm{Al}_{2} \mathrm{O}_{3}$ and will be discussed in the following.
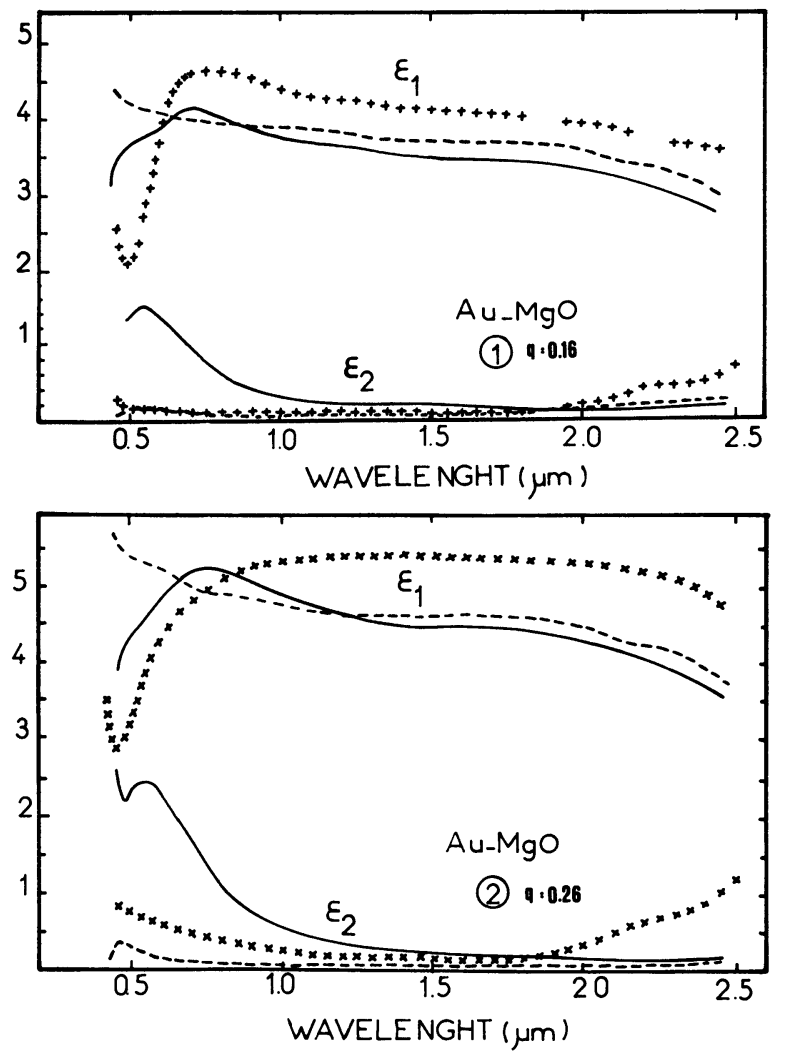
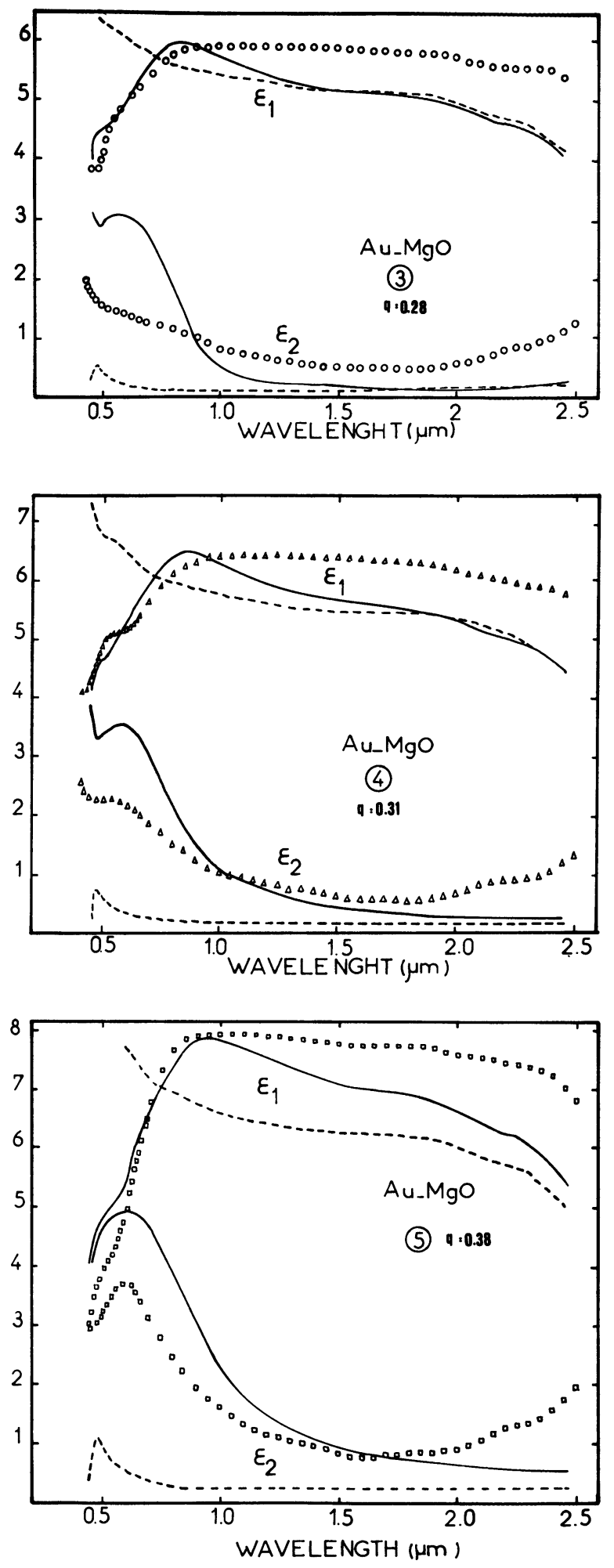

Fig. 6. - Dielectric function of Au-MgO granular thin films versus wavelength for different Au volume fractions $q$ : $(1 . q=0.16+, 2 . q=0.26 \times, 3 . q=0.280,4 . q=0.31 \Delta$, $5, q=0.38 \square$ ) see also table Ib. Fit using M.G. theory (dashed lines). Fit using BR theory (full lines).

3.2 THE EFFECTIVE MEDIUM THEORIES FITTING THE OPTICAL DIELECTRIC FUNCTION. - The dielectric function calculated in 3.1 is, in fact, an average effective 
value assuming that the composite medium is macroscopically homogeneous. The Maxwell Garnett [1] and Bruggeman [2] Effective Medium Theories (EMT) allow to calculate this effective dielectric function as a function of the volume fraction of the components and their dielectric function, in the quasistatic approximation which can be considered as valid since the optical wavelength is much more larger than the size of the particles. A modern derivation expresses the average electric field in the effective homogeneous medium as the volumic average of the fields inside the metallic and the dielectric clusters. The only difference between the two theories lies in the treatment of these two types of clusters : asymmetric in the Maxwell Garnett's approach, symmetric or selfconsistent in the Bruggeman's one; this is obvious in the formulation :

$$
\mathrm{MG} \frac{\varepsilon_{\mathrm{av}}-\varepsilon_{\mathrm{m}}}{L \varepsilon_{\mathrm{av}}+(1-L) \varepsilon_{\mathrm{m}}}=q \frac{\varepsilon_{\mathrm{i}}-\varepsilon_{\mathrm{m}}}{L \varepsilon_{\mathrm{i}}+(1-L) \varepsilon_{\mathrm{m}}}
$$

BR

$$
q \frac{\varepsilon_{\mathrm{i}}-\varepsilon_{\mathrm{av}}}{L \varepsilon_{\mathrm{i}}+(1-L) \varepsilon_{\mathrm{av}}}+(1-q) \frac{\varepsilon_{\mathrm{m}}-\varepsilon_{\mathrm{av}}}{L \varepsilon_{\mathrm{m}}+(1-L) \varepsilon_{\mathrm{av}}}=0
$$

where $q$ is the metal volume fraction, $\varepsilon_{\mathrm{i}}$ and $\varepsilon_{\mathrm{m}}$ the dielectric function of respectively the metal and the dielectric. The shape of the clusters has been introduced in these formulations via the $L$ parameter, called depolarization factor [9] as it can be directly related to the electrical polarizability of the ellipsoidal clusters assumed to have the same orientation :

$$
\alpha_{j}=\frac{\varepsilon_{\mathrm{i}}-\varepsilon_{\mathrm{m}}}{3\left[\varepsilon_{\mathrm{i}}+\left(\varepsilon_{\mathrm{m}}-\varepsilon_{\mathrm{i}}\right) L_{j}\right]} a \cdot b . c \quad j=a, b, c
$$

where $2 a, 2 b, 2 c$ denote the principal axes. In the case of an ellipsoid of revolution $(b=c)$, the depolarization factor along the principal axes can be expressed versus $c / a$ ratio (Fig. 7). When the ellipsoid is degenerated into a sphere $(c / a=1)$, we have $L_{\mathrm{a}}=L_{\mathrm{b}}=$ $L_{c}=1 / 3$ and the tensor is reduced to a scalar. For randomly oriented ellipsoids with various eccen-

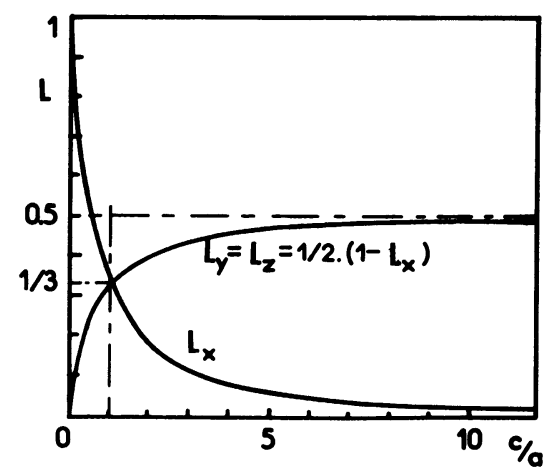

Fig. 7. - Eigenvalues of the depolarization tensor $\left(L_{x}\right.$, $\left.L_{y}, L_{z}\right)$ as a function of the excentricity $(c / a)$ of the ellipsoidal inclusion. tricity, the polarizability has to be replaced by a scalar average polarizability which cannot be related to an average depolarization factor any more. This remark is of great interest for the interpretation of $L$ fitted values.

The asymmetric expression of the Maxwell Garnett theory predicts a unique type of behaviour for the medium at all composition : either metallic if the medium is considered as dielectric inclusions in a metallic matrix, or dielectric in the reciprocal case. The self consistent Bruggeman theory with its symmetrical expression predicts on the contrary a transition between a dielectric and a metallic behaviour as the metal volume fraction $q$ increases. This threshold occurs at a critical value $q_{\mathrm{c}}$, curiously equal to $L$, i.e. directly linked to the particle shape [10].

On the other hand, the Maxwell Garnett theory predicts a sharp resonance in the average dielectric function, corresponding to the vanishing of the denominator of its explicit expression, whereas the Bruggeman average dielectric function does not diverge and presents only a broadened dumped resonance at a somewhat lower energy. These effects are attributed to the excitation of a collective mode of the conduction electrons at the metallic cluster surface. Another formulation of the same effect is the resonance of an interfacial polarization term of these clusters [12].

We now analyse the dielectric function of the $\mathrm{Au}$ $\mathrm{MgO}$ and $\mathrm{Pt}-\mathrm{Al}_{2} \mathrm{O}_{3}$ thin films in the framework of these two theories.

3.3 DiELECTRIC FUNCTIONS OF THE RESPECTIVE COMPONENTS OF THE MEDIA : $\mathrm{Au}, \mathrm{Pt}, \mathrm{MgO}, \mathrm{Al}_{2} \mathrm{O}_{3}$. The Effective Medium Theories use two structural parameters : $q$ and $L$, and two variables depending on the energy of the incident electromagnetic radiation : $\varepsilon_{\mathrm{i}}(\omega), \varepsilon_{\mathrm{m}}(\omega)$, the complex dielectric function of the metallic and the dielectric clusters in the inhomogeneous medium. The exact evaluation of $\varepsilon_{\mathrm{m}}$ and $\varepsilon_{\mathrm{i}}$ is essential for the fitting of the experimental average dielectric function $\varepsilon$ using EMT. This is a well known problem, never satisfactorily solved. We have prepared thin films of pure $\mathrm{Au}, \mathrm{Pt}, \mathrm{MgO}$ and $\mathrm{Al}_{2} \mathrm{O}_{3}$ in the same deposition conditions as the cermets. We have determined their dielectric function in the spectral range 200 to $3000 \mathrm{~nm}$ (Fig. 8) using also the same methods, and analysed their morphology. The dielectric films : $\mathrm{MgO}$ and $\mathrm{Al}_{2} \mathrm{O}_{3}$, are amorphous like in the inhomogeneous medium. MgO films exhibit a very low IR absorptivity which confirms the total oxidization of the $\mathrm{Mg}$ by reactive sputtering. The infrared absorptivity of the $\mathrm{Al}_{2} \mathrm{O}_{3}$ films which is slightly higher than the stoichiometric $\mathrm{Al}_{2} \mathrm{O}_{3}$ one, confirms the oxygen deficit of about $5 \%$ deduced from ESCA measurements. Pt films are composed of small crystallites of about $120 \AA$ therefore roughly two or three times larger than those of the Pt- $\mathrm{Al}_{2} \mathrm{O}_{3}$ films. On the other hand, the crystallite size of the Au films is ten or twenty times larger than the 70-80 $\AA$ observed in the Au-MgO films. These size differences affect essentially the part 


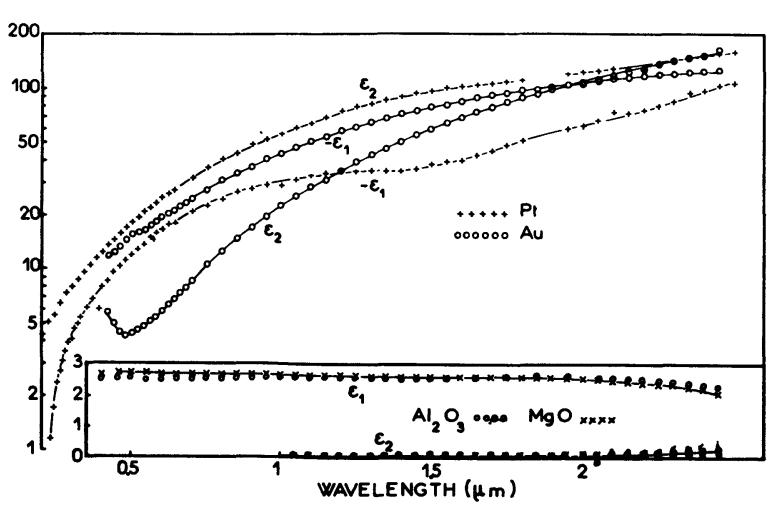

Fig. 8. - Dielectric function of pure $\mathrm{Pt}, \mathrm{Au}, \mathrm{MgO}$ and $\mathrm{Al}_{2} \mathrm{O}_{3}$ prepared in the same conditions as the granular cermet films.

of the dielectric function due to the conduction electrons $\left(\varepsilon_{\text {c.e. }}\right)$, via their relaxation time $\tau$ in the Drude formulation :

$$
\varepsilon_{\text {c.e. }}=P-\omega_{\mathrm{p}}^{2} / \omega(\omega+i / \tau) .
$$

A crystallite size dependent relaxation time $\left(\tau_{\mathrm{e}}\right)$ is classically introduced [13] by adding a corrective term to the inverse of the relaxation time in the bulk metal :

$$
1 / \tau_{\mathrm{e}}=1 / \tau_{0}+v_{\mathrm{F}} / r .
$$

This term accounts for diffuse scattering at the surface of the particle of radius $r$ of the electrons travelling with the Fermi velocity $\left(v_{F}\right)$. Quantum size effects are negligible in this spectral range.

We have neglected such a relaxation time correction in the case of $\mathrm{Pt}$ films for three reasons :

1) The particle sizes in the cermet and in the pure Pt film are not strongly different.

2) The corrective term $v_{F} / r$ presents a negligible relative value compared to the high value of $1 / \tau_{0}$ in our reference Pt film $(>0.2 \mathrm{eV})$.

The relative change in the total $\varepsilon$ due to this variation in $\varepsilon_{\text {c.e. }}$ is moreover small since the intraband contribution $\varepsilon_{\text {c.e. }}$ is not the predominant part to $\varepsilon$, the interband one $\varepsilon_{\mathrm{ib}}\left(\varepsilon=\varepsilon_{\text {c.e. }}+\varepsilon_{\mathrm{ib}}\right)$ contributing strongly to $\varepsilon$.

The same considerations applied to noble metals, and especially our pure Au films, show that the size effect correction on $\tau$ is important :

1) Due to the large crystallite size difference between pure $\mathrm{Au}$ and $\mathrm{Au}$ in $\mathrm{Au}-\mathrm{MgO}$ films.

2) Due to the low value of $1 / \tau_{0}$ in our pure $\mathrm{Au}$ films $(<0.1 \mathrm{eV})$.

3) Due to the absence of an interband transition contribution in $\mathrm{Au}$ beyond $600 \mathrm{~nm}$ [3].

Figure 9 shows the size dependent relaxation time $\hbar / \tau_{\mathrm{e}}$ for $\mathrm{Au}$ versus the particle radius. The $\hbar / \tau_{\mathrm{e}}$ value corresponding to the mean cluster size in Au-MgO films $(3$ to $5 \mathrm{~nm}$ ) is about $0.6 \mathrm{eV}$. We have tried an

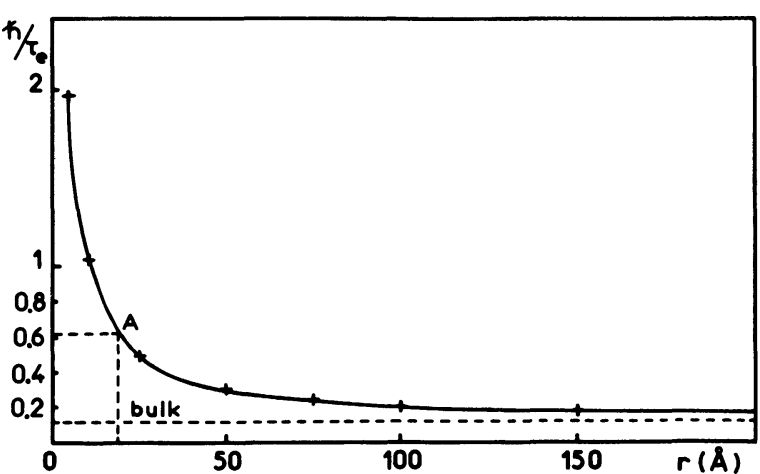

Fig. 9. - Size dependent relaxation time $\left(\hbar / \tau_{e}\right.$ in $\left.\mathrm{eV}\right)$ of conduction electrons in $\mathrm{Au}$ particles of radius $r$ (point $\mathbf{A}$ corresponds to our experiments).

other evaluation of $\hbar / \tau_{\mathrm{e}}$ by fitting the resonance in $\varepsilon$ average of Au-MgO films using BR theory, the $\hbar / \tau_{\mathrm{e}}$ being an extra adjustable parameter (as pointed out before, this resonance is very sensitive to the conduction electron parameters). The scattering of the fitted parameters is very high, but the mean value, $0.62 \mathrm{eV}$, is in very good agreement with the value deduced from the direct size observations.

\subsection{AVERAGE DiELECTRIC FUNCTION OF Au-MgO AND} Pt- $\mathrm{Al}_{2} \mathrm{O}_{3}$ : RESUl TS, FIT, DISCUSSION. - As already pointed out for the electrical conductivity, the complex dielectric function of all the five Au-MgO samples $\left(q_{\mathrm{Au}}<0.41\right)$ exhibits a dielectric behaviour (see Figs. $4: \varepsilon_{1}$ is positive and $\varepsilon_{2}$ is low in the infrared), while the cross-over between a dielectric and a metallic $\left(\varepsilon_{1}\right.$ negative and $\varepsilon_{2}$ increasing in the infrared) optical behaviour is observed in $\mathrm{Pt}-\mathrm{Al}_{2} \mathrm{O}_{3}$ films between 0.43 and $0.53 \mathrm{Pt}$ volume fraction (see Figs. 5).

The dielectric anomaly or surface plasmon resonance is clearly observed in all $\mathrm{Au}-\mathrm{MgO}$ films between 0.43 and $0.53 \mathrm{Pt}$ volume fraction (see Fig. 5). at high $\mathrm{Au}$ volume fraction. A very smooth absorption band is observed around $500 \mathrm{~nm}$ only in the first

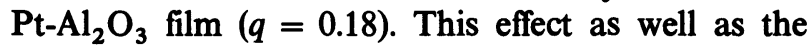
difference between the Au-MgO and $\mathrm{Pt}^{-} \mathrm{Al}_{2} \mathrm{O}_{3}$ behaviours can be at least qualitatively explained in the framework of the M.G. theory. It has been demonstrated that the M.G. function representation in the complex plane $[14,15]$ is constituted by two families of orthogonal circles, converging at a unique point corresponding to the function pole. The corresponding curves plotted for $\mathrm{Pt}$ and $\mathrm{Au}$ with various relaxation times, show that (Fig. 10) :

- The Au curve distance from the pole increases with the inverse of the relaxation time and the resonance is consequently dumped.

- The Pt curve remains far from the pole and its nearest point is shifted towards short wavelengths ( $0.42 \mu \mathrm{m}$ instead of $0.55 \mu \mathrm{m}$ for gold). The resonance is quite inexistant and equally shifted. 


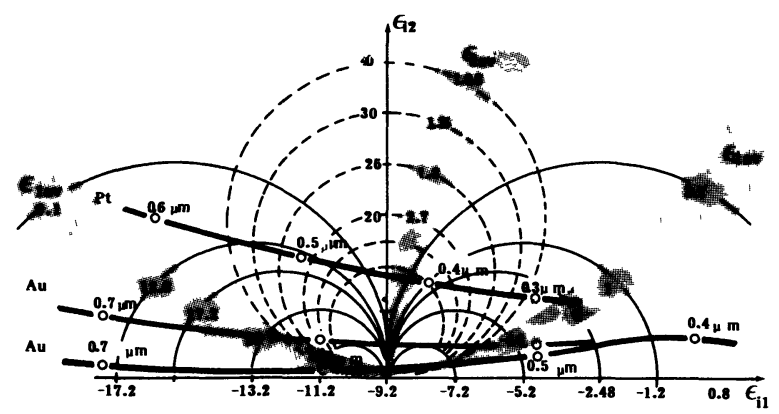

Fig. 10. - Representation in the complex plane, of the dielectric function of $\mathrm{Au}-\mathrm{MgO}$ and $\mathrm{Pt}_{-} \mathrm{Al}_{2} \mathrm{O}_{3}$ granular compounds calculated using M.G. theory with a metal volume fraction $q=0.30$. On the axis are plotted the real and imaginary parts of the metal (Au or $\mathrm{Pt}$ ) dielectric function. The (full and dashed lines) circles represent the M.G. calculated average dielectric function (real and imaginary parts) of the granular media. The intersections of the dopple full lines with the circles give, versus the wavelength, the

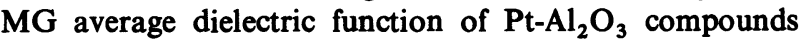
and $\mathrm{Au}-\mathrm{Al}_{2} \mathrm{O}_{3}$ compounds (for two relaxation time values).

The results of the fit of the experimental average dielectric function using the M.G. and the BR theories are plotted on figures 5 and 6 . The fitted parameters $q$ (metallic volume fraction) and $L$ (depolarization factor) are given in tables $\mathrm{I}$. In the case of $\mathrm{Pt}-\mathrm{Al}_{2} \mathrm{O}_{3}$ the fit has been performed either on $\varepsilon$ (complex) or $R$ and $T$ experimental values. It is evident on figures 5 and 6 that the M.G. theory is not adequate to account for the dielectric function of these films. The fitting procedure leads to diverging values of the depolarization factor $L$ (towards 1 or 0 ) while the fitted $q$ values remain far from the experimental ones. In contrast, the BR theory gives a fairly good account of the experimental results. It seems however more difficult to fit both the dielectric anomaly and the infrared behaviour in $\mathrm{Au}-\mathrm{MgO}$ films than the smooth variations of the dielectric function over the whole spectral range in $\mathrm{Pt}_{-} \mathrm{Al}_{2} \mathrm{O}_{3}$ films. There is no significant difference between the fit on $\varepsilon\left(\varepsilon_{1}\right.$ and $\left.\varepsilon_{2}\right)$ (Fig. 5) and on $R$ and $T$ (Fig. 4) (for $\mathrm{Pt}_{-} \mathrm{Al}_{2} \mathrm{O}_{3}$ films) except near the percolation where the fit on $\varepsilon$ is worse due to the strong variations of $\varepsilon_{1}$ compared to $R$ and $T$ at $q_{\mathrm{c}}$. A few remarks can explain these behaviours and the fit accuracy :

1) The M.G. theory which predicts a very sharp resonance at the surface plasmon frequency is evidently not adequate for these films (even for Au-MgO ones, where a resonance is observed). Moreover, even at relatively high $q$ values, M.G. always predicts very low infrared $\varepsilon_{2}$ values in the dielectric regime in contrast to our results.

2) In the M.G. and BR formulations, when the metallic inclusion $\left(\varepsilon_{\mathrm{i}}\right)$ dielectric function is large compared to the dielectric function of the average medium $(\varepsilon)$ and the dielectric matrix $\left(\varepsilon_{\mathrm{m}}\right)$, the $\varepsilon_{\mathrm{i}}$ term can be eliminated and the medium behaves now quite independently of the nature of the metallic inclusions.
This situation is reached in $\mathrm{Pt}-\mathrm{Al}_{2} \mathrm{O}_{3}$ around $500 \mathrm{~nm}$ while only around $1000 \mathrm{~nm}$ in $\mathrm{Au}-\mathrm{MgO}$, due to the quasi free electron behaviour of $\mathrm{Au}$ in the near infrared and the presence of interband transitions in Pt inducing a high near infrared dielectric function. This qualitative consideration explains the important influence of the relaxation time pointed out above in the dielectric function variations of $\mathrm{Au}-\mathrm{MgO}$ films up to $1 \mu \mathrm{m}$ and its negligible influence in the case of $\mathrm{Pt}-\mathrm{Al}_{2} \mathrm{O}_{3}$.

3) The last remark is valid except near the percolation threshold where the BR theory predicts a predominance of plasmon resonance over all wavelength (up to $\lambda \infty$ ) [10]. In fact in this critical fraction domain both $q$ and $L$ fitted values are completely inadequate, whereas they were in relatively good agreement with the experimental characteristics elsewhere (especially $L$, close to $1 / 3$ which is probably the only significant value for randomly oriented particles). In this region we will show in another paper that the BR theory fits, in fact, the quantity $q-L$, the $L$ depolarizing factor losing near $q_{\mathrm{c}}$ its significance related to the inclusion shape, as the random isolated clusters are replaced by an infinite cluster.

\section{Conclusion.}

We have presented in detail a comparative study of the structural, electrical and optical properties of $\mathrm{Au}-\mathrm{MgO}$ and $\mathrm{Pt}-\mathrm{Al}_{2} \mathrm{O}_{3}$ granular films. As predicted by the effective medium theories, we have distinguished two main phenomena in the optical properties. First, the dielectric anomaly or surface plasmon resonance which is essentially a local effect related to a resonance in the metallic clusters (depending on their nature, shape, size). We have pointed out the key role of the nature of the metal (noble or transition) on this resonance, both in the theories and in the experimental results. The influence of the size-dependent relaxation time of the conduction electrons has been discussed in Au-MgO films. Second, the optical crossover accompanying the electrical percolation and the morphological percolation at about the same critical metal volume fraction, which is essentially a structural effect related to the disorder rather than to the nature of the metallic clusters. We have shown that the experimental value of $q_{\mathrm{c}}$ is higher than the value predicted by calculations for 3D systems; this is due to the particular morphology of the films. It is well known that none of the effective medium theories gives a good account of the dielectric function around the percolation. This is obvious for M.G. which does not predict any percolation phenomenon. We have remarked that, although leading to inadequate $q$ and $L$ parameters near $q_{\mathrm{c}}$, the BR theory correctly gives the $q-L$ quantity, $L$ being no more related to the mean shape of the clusters but to the percolation critical volume fraction. This point will be developed in a further publication.

Eventually, we have shown that the near infrared 
behaviour of $\mathrm{Au}-\mathrm{MgO}$ films is more dependent on the metal dielectric function than that of $\mathrm{Pt}_{-} \mathrm{Al}_{2} \mathrm{O}_{3}$ films as the transition metal dielectric function is 3 or 4 times larger than the noble metal one, due to the contribution of interband transitions in the low energy range.

\section{Acknowledgments.}

We wish to thank C. Sella (CNRS-Bellevue) and D. Mazières (CEN-Grenoble) for sample preparation and M. L. Thèye for helpful comments and suggestions.

\section{References}

[1] Maxwell Garnett, J. C., Philos. Trans. R. Soc. London 203 (1904) 385.

[2] Bruggeman, D. A. G., Ann. Phys. (Leipz.) 24 (1935) 636.

[3] Christensen, N. E., Seraphin, B. O., Phys. Rev. B 4 (1971) 3321.

[4] Smith, N. V., Phys. Rev. B 9 (1974) 1365.

[5] Berthier, S., LAFait, J., Thin Solid Films 89 (1982) 213.

[8] Sella, C., Tran Khanh Vien, Lafait, J., Berthier, S., Thin Solid Films 90 (1982) 425.

[7] Stauffer, D., Phys. Rep. 54 (1979).

[8] Kapitulnik, A., Rappaport, M. L. and Deutscher, G., J. Physique Lett. 42 (1981) L-541.
[9] Abelès, F., Thèye, M. L., Surf. Sci. 5 (1966) 325.

[10] VAN DE Hulst, H. C., Light Scattering by small Particles (Wiley-New York) 1957.

[11] Bittard, A., Berthier, S., Lafait, J., J. Physique 45 (1984) 623.

[12] Clippe, P., Evrard, R., Lucas, A. A., Phys. Rev. B 14 (1976) 1715.

[13] Euler, J., Z. Phys. 37 (1954) 318.

[14] BerthIER, S., Thèse 3e cycle, Université P. et M. Curie, Paris 1979.

[15] Marton, J. P., Lenon, J. R., Phys. Rev. B 4 (1971) 271. 